\title{
Cochleopalpebral reflex: sensitivity and specificity in the auditory screening of newborns discharged from the neonatal intensive care unit
}

\author{
Carlos F. Martínez Cruz*, Mayra N. Ramírez-Vargas, Martina A. Guido-Campuzano, and \\ Patricia García-Alonso-Themann \\ Departamento de Seguimiento Pediátrico, Instituto Nacional de Perinatología Dr. Isidro Espinosa de los Reyes, Mexico City, Mexico
}

\begin{abstract}
Background: The sensitivity and specificity of the clinical audiological evaluation in newborns are debatable compared to neurophysiological methods of a hearing evaluation. This study aimed to determine the sensitivity and specificity of the cochleopalpebral reflex as a clinical test for hearing screening in newborns. Methods: A case-control study was designed. Newborns discharged from a neonatal intensive care unit (NICU) were included. Brainstem evoked auditory potentials were recorded. A wooden rattle was used to explore the cochleopalpebral reflex. The sensitivity and specificity of the cochleopalpebral reflex were calculated. Continuous data were analyzed with Student's $t$-test, with statistically significant $p$-values $<0.05$. Results: We selected 450 newborns who were divided into two groups: group $A$, with bilateral sensory neural hearing loss $(n=150)$, and group $B$, with normal hearing $(n=300)$. Group $A$ showed a significantly lower gestation age at birth $(p=0.005)$ compared to group $B(32.5 \pm 2.6$ vs. $34.4 \pm 3.5$ weeks). In group $A$, the cochleopalpebral reflex's sensitivity was $80 \%$ using the wooden rattle. In group $B$, the specificity was $98 \%$. Conclusions: The NICU discharged newborns' clinical hearing evaluation is not enough to exclude hearing loss. Although it may be the only diagnostic tool for hearing loss in some settings, its limitations should be considered.
\end{abstract}

Keywords: Brainstem auditory evoked potentials. Cochleopalpebral reflex. Hearing screening. Newborns. Neonatal intensive care unit.

\section{Reflejo cocleopalpebral: sensibilidad y especificidad en el tamiz auditivo de recién nacidos egresados de una unidad de cuidados intensivos neonatales}

\section{Resumen}

Introducción: La sensibilidad y la especificidad de la evaluación audiológica clínica en recién nacidos son cuestionables en comparación con los métodos neurofisiológicos de evaluación auditiva. El objetivo de este estudio fue determinar la sensibilidad y la especificidad del reflejo cocleopalpebral como prueba clínica de tamizaje auditivo en recién nacidos. Métodos: Se diseñó un estudio de casos y controles en el que se incluyeron recién nacidos egresados de una unidad de cuidados intensivos neonatales (UCIN). Se les efectuaron potenciales auditivos evocados de tallo cerebral. Para la exploración

Correspondence:

*Carlos F. Martínez Cruz

E-mail: drmtzcarlos@yahoo.com.mx
Date of reception: 06-08-2020

Date of acceptance: 29-10-2020

DOI: 10.24875/BMHIM.20000247
Available online: 08-06-2021

Bol Med Hosp Infant Mex. 2021;78(4):273-278

www.bmhim.com

1665-1146/C 2020 Hospital Infantil de México Federico Gómez. Published by Permanyer. This is an open access article under the CC BY-NC-ND license (http://creativecommons.org/licenses/by-nc-nd/4.0/). 
del reflejo cocleopalpebral se utilizó una matraca de madera. Se calcularon la sensibilidad y la especificidad del reflejo cocleopalpebral. Los datos continuos se analizaron con la prueba t de Student y se consideraron estadísticamente significativos los valores de $p<0.05$. Resultados: Se seleccionaron 450 recién nacidos y se dividieron en dos grupos: el grupo $A(n=150)$ con hipoacusia sensorineural y el grupo $B(n=300)$ con audición normal. El grupo $A$ mostró una diferencia significativa $(p=0.005)$ en cuanto a la edad de gestación al nacer en comparación con el grupo $B(32.5 \pm 2.6$ vs. $34.4 \pm 3.5$ semanas). En el grupo A, la sensibilidad del reflejo cocleopalpebral fue del $80 \%$ utilizando la matraca de madera. En el grupo B se encontró una especificidad del $98 \%$. Conclusiones: La evaluación del reflejo cocleopalpebral como prueba clínica de tamizaje auditivo en una población de recién nacidos egresados de una UCIN no es suficiente para descartar la pérdida de la audición. Aunque puede ser la única herramienta de diagnóstico para evaluar la pérdida de la audición en algunos casos, es importante considerar sus limitaciones.

Palabras clave: Potenciales evocados auditivos del tallo cerebral. Reflejo cocleopalpebral. Tamizaje auditivo. Recién nacidos. Unidad de cuidados intensivos neonatales.

\section{Introduction}

Newborns (NB) discharged from neonatal intensive care units (NICUs) are a high-risk population for developing neurological and sensory pathway sequelae, highlighting the elevated incidence of sensory neural hearing loss $(\mathrm{SNHL})^{1-6}$. The neonatal hearing screening is the most effective means of early SNHL detection. Early diagnosis and immediate intervention are decisive factors in the development and prognosis in NB${ }^{1}$.

There are two universally accepted neurophysiological methods for performing neonatal hearing screening: transient evoked otoacoustic emissions (TEOAE) (with a sensitivity of $91 \%$ and specificity of $85 \%$ ), and brainstem auditory evoked potentials (BAEP) which is the standard gold test in neonatal audiological evaluations ${ }^{1}$. These neurophysiological methods for neonatal hearing screening, with high sensitivity and specificity, ensure that all suspected cases of hearing loss are identified in the first phase and then confirmed in subsequent phases ${ }^{1,7}$.

The sensitivity and specificity of clinical audiological evaluations in NB and infants are debatable ${ }^{8}$, especially with neurophysiological hearing evaluation methods. However, a hearing evaluation with neurophysiological methods without clinical correlates is incomplete.

The cochleopalpebral reflex (CP-R) was first reported through auditory stimulation in NB by Preyer in $1882^{9}$. It consists of an involuntary blink of the eye caused by the orbicularis oculi muscle contraction in response to sounds of high pressure. This reflex can be intentionally evoked by the clinician and represents the first stage of auditory behavioral observation for NB and young infants ${ }^{10-17}$. The clinical examination of the CP-R in NB is barely used today and has been replaced by neurophysiological hearing tests in NB. The normative committee for infant hearing loss, the Joint Committee on Infant Hearing, do not include this first stage of auditory behavioral observation in the NB. Conversely, it initiates clinical auditory evaluations from the second stage of auditory behavioral observation, starting at 6 months of age using the distraction audiometry".

This study aimed to determine the sensitivity and specificity of the cochleopalpebral reflex as a clinical auditory screening test in NB discharged from NICU.

\section{Methods}

We designed a case-control study with the following inclusion criteria: NB discharged from a NICU in Mexico City, born from January 1, 2008, to December 30, 2018, regardless of their morbidity during their NICU stay, gestational age, and birth weight. For comparison and analysis purposes, two groups were established: NB with bilateral sensory neural hearing loss (group $A$ ) and NB with normal hearing (group B).

We considered the following conditions as exclusion criteria: congenital head and neck malformations, children with conductive hearing loss, children with auditory neuropathy spectrum disorder ${ }^{5}$, and NB with unilateral hearing loss.

\section{Brainstem auditory evoked potentials}

All the NB included in the study underwent brainstem auditory evoked potentials (BAEP) determinations using the international ten-twenty system ${ }^{18}$ at hospital discharge (2 months of age), and a second confirmatory determination at 6 months of chronological age, using a Nicolet Viking Quest computer (Nicolet Biomedical Inc, Madison State, WI).

The test was performed in a quiet room reserved for this purpose and sleeping NB. Cerebral electrical 
activity was recorded using gold disc electrodes, placed with previous alcohol-acetone cleaning, and application of conductive gel with the following assembly $A 1-C z$, A2-Cz, FPZ ground ( $\mathrm{Cz}$ cranial vertex, A1 and A2 both mastoid processes). The studied ear was (-), and the contralateral was ground.

The impedance of the electrodes was kept below $4 \mathrm{k} \Omega$. The bandpass filters were placed between 300 $3000 \mathrm{~Hz}$. Post stimulation analysis time was $10 \mathrm{~ms}$. BAEP determinations were performed by monaural click-type stimulation. Initially, at an intensity of 30 decibels normal hearing level $(\mathrm{dB} \mathrm{nHL})$, and gradually increasing to $80 \mathrm{~dB} \mathrm{nHL}$. The contralateral ear was masked with white noise at $40 \mathrm{~dB} \mathrm{nHL}$ below the stimulus intensity with a frequency of $11.4 \mathrm{~Hz}$. We applied 1500 stimuli with their respective replication in rarefaction mode and searched the auditory threshold. The stimulus duration was $100 \mu \mathrm{s}$; the clicks were released through TDH-49P headphones (Telephonics Co., Huntington, NY).

Normal peripheral hearing sensitivity was considered when the response at $40 \mathrm{~dB} \mathrm{nHL}$ or less for each ear provided positive reproducibility of the $\mathrm{V}$-wave. Hearing thresholds equal to or greater than $45 \mathrm{~dB} \mathrm{nHL}$ was the $\mathrm{SNHL}$ criterion. Results at 6 months of age were taken as a diagnostic test.

\section{Cochleopalpebral reflex}

The cochleopalpebral reflex (CP-R) was explored in all the NB included in the study during the first trimester of extrauterine life in the first audiological consultation.

For the CP-R examination, an $18 \times 5 \mathrm{~cm}$ wooden rattle was used. This rattle produces high-pitched sounds of $85 \mathrm{~dB} \mathrm{nHL}$ at a distance of $60 \mathrm{~cm}$. The loudness was measured with a portable sound level meter [Model 2250, BZ-7222. ISO 7196: 1995. ANSI S1.422001 (R2011). Brüel \& Kjær Sound \& Vibration Measurement A/S. DK-2850 Nærum, Denmark] in an isolated $3 \times 3 \mathrm{~m}$ sound chamber.

The rattle was rotated at a distance of approximately $60 \mathrm{~cm}$ in front of the NB face. The NB should be calm, awake, not crying, and preferably with no movements to distinguish the behavioral changes practically at the turning of the rattle (Figure 1).

Three attempts were made to search for a positive response with the presence of CP-R. The presence or absence of CP-R was recorded. Two certified audiologists performed the evaluations in all the cases included in the study.

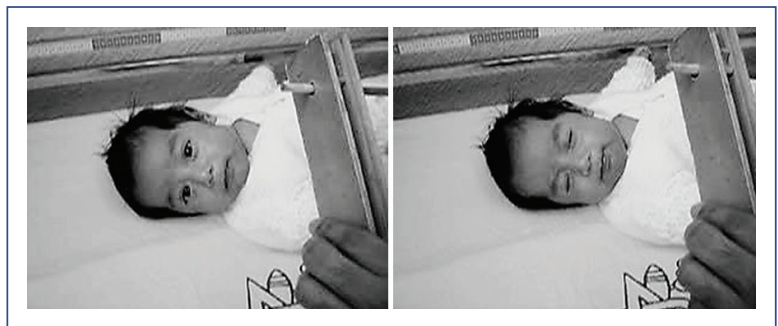

Figure 1. Cochleopalpebral reflex evoked by a wooden rattle.

One of the advantages of this toy is that it does not require large movements or efforts to cause noise, enabling the audiologist to concentrate and observe the NB during the exploration.

Clinical audiological evaluations with the CP-R and BAEP were performed with a double-blind technique. The persons who carried out the BAEP were unaware of the clinical test results, and the clinicians who carried out the CP-R were unaware of the results of BAEP.

Parents were informed of the importance of the audiological follow-up, the study purposes, and the research benefits. The signed informed consent form was requested based on the institute's research committee and the Helsinki declaration.

\section{Statistical analysis}

Continuous data were presented as means and standard deviations and analyzed using the Student's $t$-test. Categorical variables were presented as frequencies and percentages and analyzed using the $\chi^{2}$ test; $p$-values $<0.05$ were considered statistically significant. We calculated the sensitivity and specificity of the CP-R and determined the positive and negative predictive values of the test.

For data analysis, we used the statistical program IBM SPSS for Windows version 20.0 (IBM Corp., Armonk. NY, USA).

\section{Results}

During 10 years (from January 2008 to December 2018), we selected 450 NB discharged from a NICU. The mean chronological age was $2 \pm 1$ months. They were divided into two groups: group $A, N B$ with a diagnosis of bilateral SNHL $(n=150)$, and group $B, N B$ with normal bilateral hearing $(n=300)$. The frequency of bilateral SNHL in this sample was $33 \%$. 
Table 1. General characteristics of both groups

\begin{tabular}{|l|c|c|c|c|c|c|c|}
\hline Group & \multicolumn{3}{|c|}{$\begin{array}{c}\text { A (SNHL) } \\
\text { (n= 150) }\end{array}$} & \multicolumn{3}{c|}{ B (NH) } \\
(n=300)
\end{tabular}

*Student's t-test.

$\mathrm{NH}$, normal hearing; n, frequency; SNHL, sensory neural hearing loss; SD, standard deviation.

Statistically significant differences between group A and group $B$ were observed regarding the gestational age at birth (32.5 \pm 2.6 vs. $34.4 \pm 3.5$ weeks; $p=0.005)$, birth weight (1530 \pm 581 vs. $1723 \pm 805 \mathrm{~g} ; p=0.01$ ), and hospital stay (58 \pm 41 vs $31 \pm 22$ days; $p=0.001$ ) (Table 1).

No statistical difference was observed regarding sex $(p=0.06): 56 \%(n=84)$ and $44 \%(n=130)$ of the NB were male in group $A$ and group $B$, respectively.

In group $A(S N H L)$, the sensitivity of the CP-R was $80 \%$ using the wooden rattle, and in group $\mathrm{B}(\mathrm{NH})$, the specificity was $98 \%$ (Table 2 ).

In group $A$, the BAEP results showed mean hearing thresholds at 90 decibels in both ears, unchanged at 2 and 6 months of age. Ninety-eight cases (65.3\%) showed no bilateral response at $100 \mathrm{~dB} \mathrm{nHL}$. Ten cases in this group $(6.7 \%)$ showed a $\mathrm{V}$ wave of the BAEP at $95 \mathrm{~dB} \mathrm{nHL}$. Forty-two (28\%) showed hearing thresholds between 45 and $90 \mathrm{~dB} \mathrm{nHL}$.

In group $\mathrm{B}, \mathrm{BAEP}$ studies showed hearing thresholds at $35 \mathrm{~dB} \mathrm{nHL}$ at 2 months of age and $29 \mathrm{~dB} \mathrm{nHL}$ at 6 months of age (Table 3).

\section{Discussion}

The main objective of hearing screening is that NB can receive an audiological evaluation during the first month of age to confirm hearing loss diagnosis and initiate auditory habilitation before 6 months of age.

In the present day, there are high sensitivity and specificity neurophysiological studies that make hearing screening at early ages easy and accessible to the general population. However, screening needs specialized equipment and personnel. Exploring hearing in NB and infants does not need to be the domain of the audiology specialist since the procedure is simple, easy, feasible, replicable, and inexpensive, as shown in the present study. The balance between neurophysiological
Table 2. Sensitivity and specificity of the cochleopalpebral reflex in newborns discharged from a neonatal intensive care unit

\begin{tabular}{|c|c|c|c|}
\hline Group & $\begin{array}{c}\text { A (SNHL) } \\
\mathbf{n}(\%)\end{array}$ & $\begin{array}{c}\text { B (NH) } \\
\mathbf{n}(\%)\end{array}$ & $\begin{array}{c}\text { Total } \\
\mathbf{n}(\%)\end{array}$ \\
\hline CP-R & & & \\
Absent & $121(27 \%)$ & $5(1 \%)$ & $126(28 \%)$ \\
Present & $29(6 \%)$ & $295(66 \%)$ & $324(72 \%)$ \\
Total & $150(33 \%)$ & $300(67 \%)$ & $450(100 \%)$ \\
& $\begin{array}{c}\text { Sensitivity } 80 \% \\
\text { Positive }\end{array}$ & $\begin{array}{c}\text { Specificity } 98 \% \\
\text { Negative }\end{array}$ & \\
& predictive value & predictive value & \\
& $96.03 \%$ & $91.04 \%$ & \\
& & &
\end{tabular}

CP-R, cochleopalpebral reflex; $n$, frequency; $N H$, normal hearing; SNHL, sensory neural hearing loss.

Table 3. Brainstem auditory evoked potentials thresholds in discharged newborns from the neonatal intensive care unit

\begin{tabular}{|l|c|c|c|c|}
\hline Group & \multicolumn{2}{|c|}{ A (SNHL) } & \multicolumn{2}{c|}{ B (NH) } \\
\hline Variable & $\mathbf{n}$ & Mean \pm SD & $\mathbf{n}$ & Mean \pm SD \\
\hline $\begin{array}{l}\text { At 2 months of age } \\
\text { Right ear } \\
\text { Left ear }\end{array}$ & 150 & $90.7 \pm 15.9$ & 300 & $35.8 \pm 10.5$ \\
\hline $\begin{array}{l}\text { At 6 months of age } \\
\text { Right ear } \\
\text { Left ear }\end{array}$ & 150 & $91.7 \pm 14.2$ & 300 & $36.2 \pm 11.3$ \\
\hline
\end{tabular}

Values in decibels.

$\mathrm{NH}$, normal hearing; $\mathrm{n}$, frequency; SD, standard deviation; SNHL, sensory neural hearing loss.

studies and clinical practice in evaluating NB and infant hearing will have correlates that complement each other, establishing a complete practice in pediatric audiology. The intentional exploration of the CP-R to evaluate NB audiological status does not intend to defer the neurophysiological hearing test but complement a 
comprehensive audiological evaluation where the clinical correlate and the neurophysiological hearing findings will lead to the best diagnostic and therapeutic decisions. In situations where there is no access to neurophysiological hearing tests for the NB, a clinical hearing evaluation such as the one proposed here would be better than no test.

The general characteristics of this NB population discharged from NICU stand out for the group with SNHL (lower gestational age and birth weight, and more extended stay in the NICU) compared with the group with normal hearing. These observations represent a greater susceptibility to hearing damage in a critical period of high vulnerability, such as premature birth and low birth weight. These indicators and audiological risk factors in NICU graduates have been extensively documented and discussed in the previous publications ${ }^{6}$.

The sensitivity of the CP-R was $80 \%$, and the specificity was $98 \%$ using a wooden rattle as a clinical test for hearing screening in a population of NB discharged from a NICU in Mexico City. With these results, the clinical hearing evaluation of NICU discharged NB is not sufficient to discard hearing loss and should be complemented with neurophysiological hearing tests.

Poblano et al. reported a $38 \%$ sensitivity of the CP-R and a specificity of $97 \%$ in a study with a sound toy test and CP-R in the early detection of hearing loss in infants ${ }^{8}$.

The physiology of CP-R is not yet fully understood; however, it is known that the afferent signal travels through the cochlear nerve at the level of the brainstem, annular protuberance, including the mesencephalic reticular formation, the nucleus of the lateral lemniscus, and the inferior colliculus, and the facial nerve transmits the efferent information to the final organ (the eyelids).

High sound pressure of approximately $80 \mathrm{~dB} \mathrm{nHL}$, reached in milliseconds, is necessary for exploring the $\mathrm{CP}-\mathrm{R}$ in NB and infants since lower sound intensities may not trigger the reflex.

For this reason, we suggest high sound pressure toys such as the wooden rattle, a triangle, drum, or bongo. Amiel-Tison and Grenier used loud applause in front of the infant to trigger this reflex, which may also be help$\mathrm{fu}^{19}{ }^{19}$. Regardless of the sound method, the clinician's knowledge and experience are of paramount importance to determine normal or abnormal hearing in the newborn or infant at the time of evaluation. If two or more observers are added to this auditory behavioral observation and all reach a uniform criterion, the test will have greater validity. The procedure can also be filmed for later evaluations.

To date, there is no standardization or uniformity for the use of these sound instruments, so they are used under clinical evaluation. They can be measured with sound level meters, but they are difficult to calibrate.

If the infant arrives asleep to the evaluation, it will offer a good moment to evaluate, as long as the sleep is in a superficial phase. The infant's eyelashes should be touched to determine if the sleep is superficial or deep. If the NB strongly closes the eyelids or shows discomfort movements, it indicates superficial sleep, and the healthcare personnel can perform the audiological evaluation. On the contrary, if the infant is not bothered, then the sleep is in a deep phase, and the test is not recommended. The auditory responses observed during shallow sleep are practically the same as those mentioned previously. The CP-R will show more significant lid closure and may result in the infant waking up.

As could be expected, this method has limitations. An evaluation with negative CP-R does not guarantee that the NB or infant has hearing damage, but it indicates a suspect until proven otherwise. Therefore, in these cases, it is suggested to attend the audiological clinic for a BAEP determination. A positive reaction will pass the hearing screening as good.

Infants with unilateral hearing loss, superficial or medium degree of hearing loss will pass this evaluation modality. The false negatives in the present study (29 cases, $20 \%$ of group A) are justified for being cases with medium stage SNHL (documented with BAEP).

Face muscles must be relaxed to explore this auditory reflex in the NB. Therefore, a child crying cannot be evaluated. As the infant becomes older than 6 months, it will inhibit or control this reflex at the cortical level: at the first twist of the rattle, the CP-R is shown, but the reflex becomes weaker in the second and third tries. Some infants with neurological or visual damage may lack CP-R inhibition when faced with this noise evocation.

In NB, the blink reflex can also be caused by photo-stimulation and used to evaluate visual pathways and part of the function of the brain stem in this modality ${ }^{13,14}$.

BAEP represents the neurophysiological test of choice in NB discharged from NICU and the standard gold test for the evaluation of hearing in $\mathrm{NB}^{1,7}$. NB with normal hearing will have average BAEP results, and NB and infants with SNHL will have BAEP without bilateral response or only $\mathrm{V}$ wave at $95 \mathrm{~dB} \mathrm{nHL}$. 
The case-control design is a limitation of the study; a cohort study should be designed to include the entire population, with no exclusion criteria.

The clinical hearing evaluation in NICU-discharged NB is not enough to exclude hearing loss. Although it may be the only diagnostic tool for hearing loss in some settings, it is important to consider its limitations.

\section{Ethical disclosures}

Protection of human and animal subjects. The authors declare that no experiments were performed on humans or animals for this study.

Confidentiality of data. The authors declare that they have followed the protocols of their work center on patient data publication.

Right to privacy and informed consent. The authors have obtained the written informed consent of the patients or subjects mentioned in the article. The corresponding author has this document.

\section{Conflicts of interest}

The authors declare no conflict of interest.

\section{Funding}

None.

\section{References}

1. American Academy of Pediatrics, Joint Committee on Infant Hearing. Year 2007 position statement: principles and guidelines for early hearing detection and intervention programs. Pediatrics. 2007;120:898-921.
2. Abdullah A, Dahari KASA, Tamil AM, Rohana J, Razif MYM, Shareena I. The prevalence of hearing loss among babies in the neonatal intensive care unit in a tertiary hospital in Malaysia. Medeni Med J. 2020; 35:116-20.

3. Jayagobi PA, Yeoh A, Hee KYM, Sok Bee Lim L, Choo KP, Kun Kiaang HT, et al. Hearing screening outcome in neonatal intensive care unit graduates from a tertiary care centre in Singapore. Child Care Health Dev. 2020;46:104-10.

4. Chang J, Oh SH, Park SK. Comparison of newborn hearing screening results between well babies and neonates admitted to the neonatal intensive care unit for more than 5 days: analysis based on the national database in Korea for 9 years. PLoS One. 2020;15:e0235019.

5. Xu J, Weng M, Li N, Wu X, Gao L, Yao H, et al. Relationship research between auditory neuropathy spectrum disorder and exchange transfusion in neonates with severe hyperbilirubinemia. Int J Pediatr Otorhinolaryngol. 2019:123:146-50.

6. Martínez-Cruz CF, Poblano A, Fernández-Carrocera LA. Risk factors associated with sensorineural hearing loss in infants at the neonatal intensive care unit: a 15-year experience at the National Institute of Perinatology (Mexico City). Arch Med Res. 2008;39:686-94.

7. Secretaría de Salud. Programa de acción específico 2007-2012. Tamiz auditivo neonatal e intervención temprana. Ciudad de México: Secretaría de Salud; 2009.

8. Poblano A, Chayo I, Ibarra J, Rueda E. Electrophysiological and behavioral methods in early detection of hearing impairment. Arch Med Res. 2000;31:75-80.

9. Preyer W. [Die Seele des Kindes]. Leipzig: Th. Grieben's Verlag; 1882.

10. Hopf HC, Bier J, Breuer B, Scheerer W. Cochleo-palpebral reflex, a test method for objective (reflex) audiometry. Arch Klin Exp Ohren Nasen Kehlkopfheilkd. 1972;201:147-58.

11. Motta G, Facchini GM, D'Auria E. Objective conditioned-reflex audiometry. Acta Otolaryngol Suppl. 1970;273:1-49.

12. Yamada A. Blink reflex elicited by auditory stimulation: clinical study in newborn infants. Brain Dev. 1984;6:45-53

13. Yasuhara A, Yamada A, Matsumura T. Photoevoked eyelid micro-vibration (blink réflex elicited by flash stimuli) in newborns and children. Brain Dev. 1983;5:474-7.

14. Yasuhara A, Yamada A, Sugimoto T, Matsumura T. Studies on photopalpebral reflex in neonatal intracranial hemorrhage and neonatal asphyxia. Brain Dev. 1983;5:159.

15. Maggi CR, Pacheco LC, Tochetto T, Gonçalves MS, Pedroso FS. Habituation of the blink reflex in the neonatal period and development of auditory processing. Einstein (São Paulo). 2011;9:31-5.

16. Lowe A. Audiometría en el niño: implicaciones pedagógicas. Buenos Aires: Editorial Médica Panamericana S.A.; 1981.

17. Notherm JL, Downs MP. Pruebas audiológicas clínicas. La audición en Ios niños. Madrid: Editorial Salvat; 1981

18. Klem GH, Lüders $\mathrm{HO}$, Jasper $\mathrm{HH}$, Elger $\mathrm{C}$. The ten-twenty electrode system of the International Federation. The International Federation of Clinical Neurophysiology. Electroencephalogr Clin Neurophysiol Suppl. 1999:52:3-6.

19. Amiel-Tison $\mathrm{C}$, Grenier A. Vigilancia neurológica durante el primer año de vida. Barcelona: Masson; 1988. 